\title{
On Cruise Tracks for a Global Census of Marine Life
}

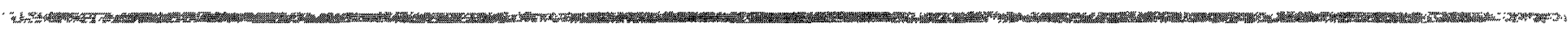

John F. Caddy and F. Carocci

United Nations Food and Agriculture Organization - Rome Italy

\begin{abstract}
A procedure is proposed for reconciling different scientific priorities within a multi-objective global survey in which the needs of individual participants, their preferred sampling areas and methods, and requirements for treatment and storage of samples, all have to be considered in deciding a common cruise schedule and work plan. The paper proposes a decision-making framework that will help reconcile the individual requirements of each scientific program and its differing objectives within the joint endeavor, through an objective exercise. Negotiation on priority survey areas and stations would be facilitated and focussed by objective information compilation. Priorities of the different parties as to areas to be surveyed can be expressed in the form of separate maps for each program. These can be reconciled within a Geographic Information System (GIS) format, after weighting each by objective criteria ideally prepared by an independent review committee. The combined overlays for each program can be used as a basis for stratified station allocation. The weighting allocated to each of the participating scientific programs should reflect inter alia the degree to which each program's requirements are in accordance with overall objectives, areas of operation, survey vessel procedures and the joint work of the expedition. The role of historical data in optimizing the impact of the Census of Marine Life, and the needs for the Census to provide adequate baselines for future work, are also discussed.
\end{abstract}

\section{Introduction}

The concept of carrying out a global survey of living ocean resources has been raised recently, and practical steps have since led to convening of several meetings. These attempted to define the multiple objectives and scope of what will probably prove one of the more ambitious such programs to date: a program which will be attempting to survey living resources of some $2 / 3$ of the planet's surface. If successful, it perhaps, in the future will be considered comparable in significance to the Challenger surveys carried out roughly a century ago.
The meetings to date make it clear that we are dealing with an endeavor in which a number of subject matter experts may differ considerably. This will be true not only with respect to the type of observations they wish to carry out at each station, the equipment and sample storage requirements, but also in the ocean areas which they believe should receive priority for attention. Evidently, these potentially conflicting requirements for sampling may prove a major obstacle for cruise design, and what is proposed here is to explore a systematic approach to combining multiple objectives. The approach is similar to that proposed by Caddy (1996) in the case of shared stock negotiations; namely, to reduce the requirement for negotiating to that of discussing the weighting factors to be assigned to each individual proposal. A GIS overlay technique will be used to reconcile different objectives, to the extent that this is possible.

\section{The problem}

The combination of the large geographical scope (hence high cost in ship time for those observations requiring a sea surface platform), and the multiple sampling requirements at each station, pose problems in reconciling multiple sampling priorities. Different biota require differing sampling gear/storage and at-sea analyses, and these will have to be reconciled between those multiple "parties" contributing to the joint endeavor. Clearly, what will result is a cruise track that will not be ideal for any one party. What we are looking for then, is a "least worst" solution that can be accepted by all concerned. This solution might also take into account the financial contribution each scientific program makes to the joint endeavor, (not considered further here, though not incompatible with the approach proposed). It should also lead to the selection of the oceanic sampling sites which are of common interest to as many participants as possible.

The problems of stratification/variance estimation were highlighted at the Southampton meeting (see synopsis by Bannister, this volume). First, existing data may provide an incomplete basis for any stratification 
system or sampling, given that we have little information in many cases to form the basis for such a stratification. Subject matter specialists will however presumably have areas of high intrinsic interest that differ from those of other parties. Using a fixed grid of samples at regular intervals when the total number of stations will have to be seriously limited by the vast distances to be covered, risks resulting in a large proportion of stations yielding relatively low or uniform results for a considerable expenditure of ship time. This approach will likely miss areas that specialists have identified as of particular interest. There seems, in our opinion, therefore, little choice but to allow each subject matter specialist to prepare their individual ideas for optimal coverage based on information or expert intuition, and then find some way of reconciling these visions. The risk with all "mixed" endeavors is of course that estimates of sampling variance will be sub-optimal and what will result risks becoming a hugely expensive specimen collecting trip a la Ed Ricketts of the "Sea of Cortez" fame. This will cause future scientists endless frustration in comparing outputs with the Census results.

No definitive solution to the problem of variance estimation is proposed here, though the approach has elements of a stratified approach that could be built upon. It is suggested however that if common criteria for stratification cannot be agreed to (and this seems likely, given the multiple objectives), post stratification might be performed on the stations chosen following the compromise procedure suggested here, or individual stations could be converted into local clusters or sub-station replicates. This second possibility would have the advantage of allowing some local deployment of slowacting sampling devices, or of time consuming on-site observations of species interactions etc. (Presumably, of course, towed sensors would be deployed between the $1000 \mathrm{~km}$ or more separating such a "mega-station" or "work area" from the next such site in line).

\section{The Historical Record}

At the Southampton meeting it seemed to have been agreed that the early phase of the "Census" would involve data mining, and discrimination of historical data to reject sets of data believed to be seriously flawed. Such sets of data, especially if spatially located, could form a contribution through GIS procedures to an oceanic atlas of historical information; either as spatially distributed "benchmarks" at a particular point in time, or as time series for a point or area in space.

Figure 1 attempts to illustrate that comparisons of the census data will first be possible with historical data that could reveal important differences or possible trends. Whether the significance of the differences revealed for all types of observations cannot be guaranteed within such a multiple framework proposed is seen as an inevitable consequence of a multiple objective endeavor. Hopefully however, using a cluster sampling approach in areas previously surveyed, will yield some criteria that will allow comparison/or even just calibration with earlier data sets, and point up possible changes for further investigation. For areas/species/sampling methods not yet explored, the problem of comparability will of course be postponed to when bits or the entire Census are repeated in future years. Inevitably in this case, given that future technologies are expected to be sophisticated in ways we cannot now imagine, our ability to forecast the approaches used in the future are likely to be rather defective. One response, almost inevitably, will largely consist of taking sample replicates at each station, and preserving/storing the samples with minimal treatment: inevitably a bulky storage problem that will need to be taken into account in a multi-objective endeavor. This will involve a major financial commitment both on-ship and on shore, but more to the point, sampling and storage requirements on board may not be easily reconciled.

As noted, it is supposed that historical data sets will be used by each scientific "party" in different ways to shape the questions they will ask in the Census. Here it should be specified that a "party" consists of a group of scientists sharing similar interests by taxonomic group or discipline, who are expected to have reconciled their internal differences before negotiating with the other "parties" involved in the multidisciplinary activity.

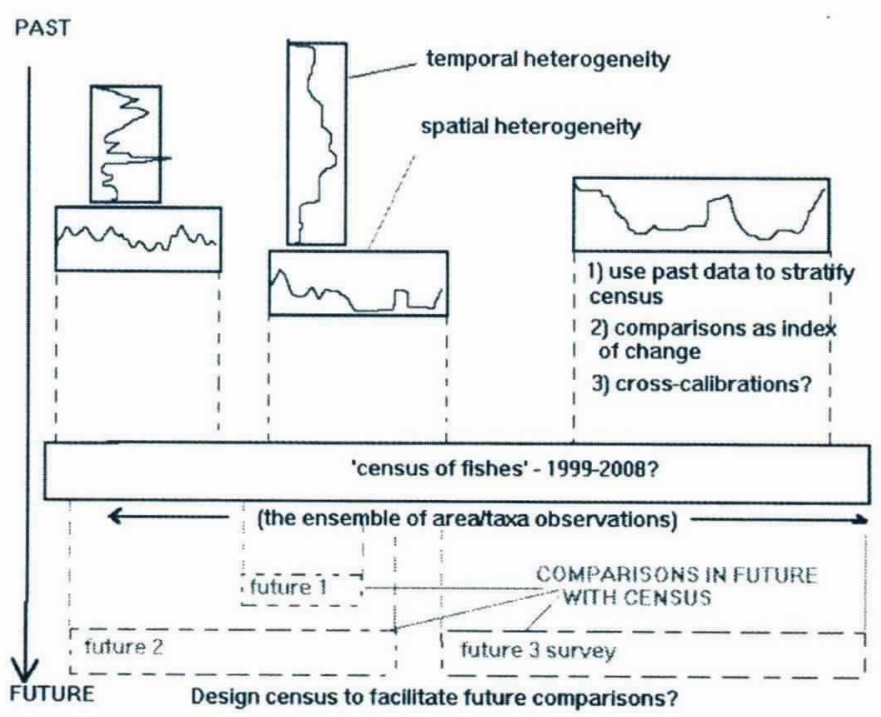

Figure 1: Illustrating the potential uses of the census as a baseline for measuring change. Appropriate historical data can be compared with the census if this takes them into account. This data set will be used by future scientists for comparative studies. 


\section{Methods}

In the earlier paper mentioned above, Caddy (1996) suggested that friction can be reduced in reconciling different approaches, if each party to a negotiation agrees to divide the process into:

1. Formulation of individual positions (in this case provision of a map showing perhaps, high, medium, and low areas of interest to each party)
2. A commonly applied listing of the practical contribution of the party to the common endeavor or its compatibility with other approaches should be prepared. Here one could also include the financial contribution each makes to the cruise (though this is not considered further in the following example). Conversely, the disadvantages that each approach poses, given severe time/space/cost constraints (especially on-ship), to the common endeavor

\section{TABLE 1:}

A possible approach to providing a ranking for hypothetical observation types proposed by parties $A$ to $E$ of a multi-disciplinary survey.

\begin{tabular}{|c|c|c|c|c|c|c|c|c|c|}
\hline PARTY/SAMPLING (GEAR) & $(1)^{*}$ & (2) & (3) & (4) & (5) & (6) & etc & RANK SUM & WEIGHTING \\
\hline A) Benthos (grab) & 3 & 1 & 2 & 2 & 2 & 2 & & 12 & 0.112 \\
\hline A) Benthos (core) & 3 & 2 & 3 & 1 & 2 & 2 & & 13 & 0.121 \\
\hline B) Nekton (gear 1) & 1 & 2 & 3 & 2 & 1 & 1 & & 10 & 0.093 \\
\hline B) Nekton (gear 2) & 2 & 3 & 3 & 2 & 2 & 1 & & 13 & 0.121 \\
\hline \multicolumn{10}{|l|}{ C) Mesopelagic fish } \\
\hline (midwater trawl) & 3 & 1 & 3 & 3 & 1 & 2 & & 13 & 0.121 \\
\hline C) Hydroacoustic & 3 & 3 & 1 & 2 & 3 & 1 & & 13 & 0.121 \\
\hline D) Cephalopods (gear 1) & 2 & 2 & 2 & 1 & 2 & 2 & & 11 & 0.103 \\
\hline D) Cephalopods (traps) & 1 & 2 & 3 & 1 & 1 & 2 & & 10 & 0.093 \\
\hline E) Mammal observation & 3 & 3 & 1 & 1 & 3 & 1 & & 12 & 0.112 \\
\hline TOTALS & 21 & 19 & 21 & 15 & 17 & 14 & & 107 & \\
\hline
\end{tabular}

*KEY:
1) $1 /$ (time spent sampling per station)
4) $1 /$ (steaming time to preferred areas)
2) $1 /$ (bulk storage requirements)
5) compatability with other observation types
3) $1 /$ (lab time for processing on station)
6) cost contribution

TABLE 2:

'Hits' from the procedure for each of the high and medium priority areas proposed by each party in fig 3

Low Priority

No. samples Surface $(\mathrm{sq} \mathrm{km})$
Medium Priority

No. samples Surface (sq km)
High Priority

No. samples Surface (sq km)
Challenger

Deep Ocean

11

34

Historical importance

Mid Atlantic Ridge 13

Atlantic Transects

38

Continental Shelf
9
$3.8 \mathrm{e}+06$

$7.3 \mathrm{e}+06$

$5.8 \mathrm{e}+06$

$6.0 \mathrm{e}+06$

$3.0 \mathrm{e}+06$
2

4

4

11
$1.9 \mathrm{e}+05$

$4.8 \mathrm{e}+05$

5

$1.2 e+06$

$1.1 e+06$

$4.0 \mathrm{e}+06$

40

$5.6 \mathrm{e}+06$ 
should also be provided in some ranked form. This aspect could be registered as a spreadsheet such as that in Table 1 which shows some purely hypothetical considerations. The valuations given for each party could be semi-quantitative, or be the best judgements by a hypothetical impartial planning group. Figure 2 illustrates the potentially iterative nature of the proposed procedure.

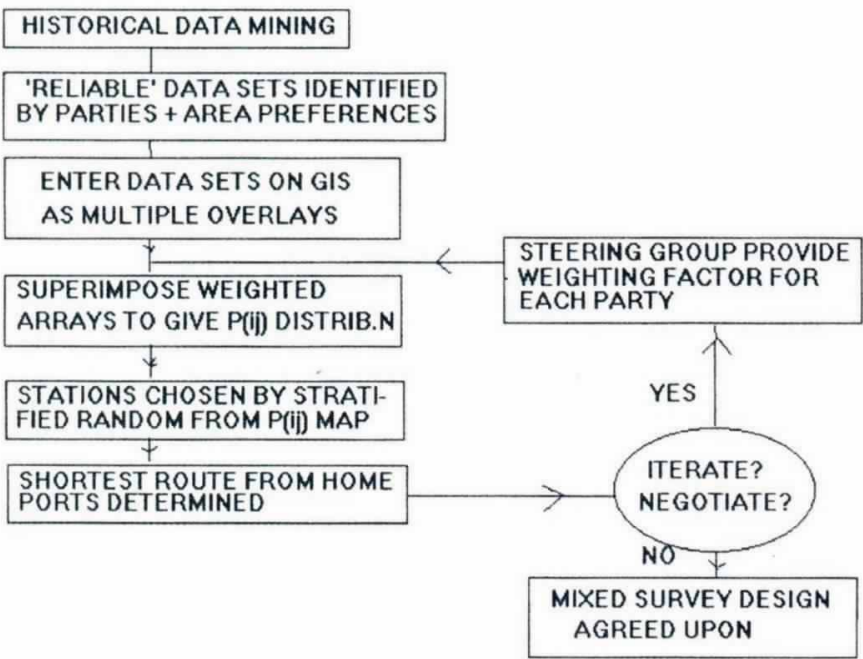

Figure 2: Illustrating a procedure for negotiating a joint cruise design in light of individual requirements by each party.

3. For any given unit area (however defined) on this global oceanic map, the probability of a station falling there would be given by:

$$
P_{i j}=\sum_{k}\left({ }_{k} D_{i j} * W_{k}\right)
$$

where:

${ }_{k} D_{i j}$ is the preference by party $k$ for working in lat-long unit $i, j$

$\mathrm{W}_{\mathrm{k}}$ is the weighting resulting from a process such as that illustrated in Table 1 (as noted, for fairness, individual weighting should be estimated by an independent and unbiased steering group).

4. Negotiations could be carried out to modify the weightings.

In theory, stages 1 to 4 could form a loop and be repeated until some suitable compromise is arrived at.

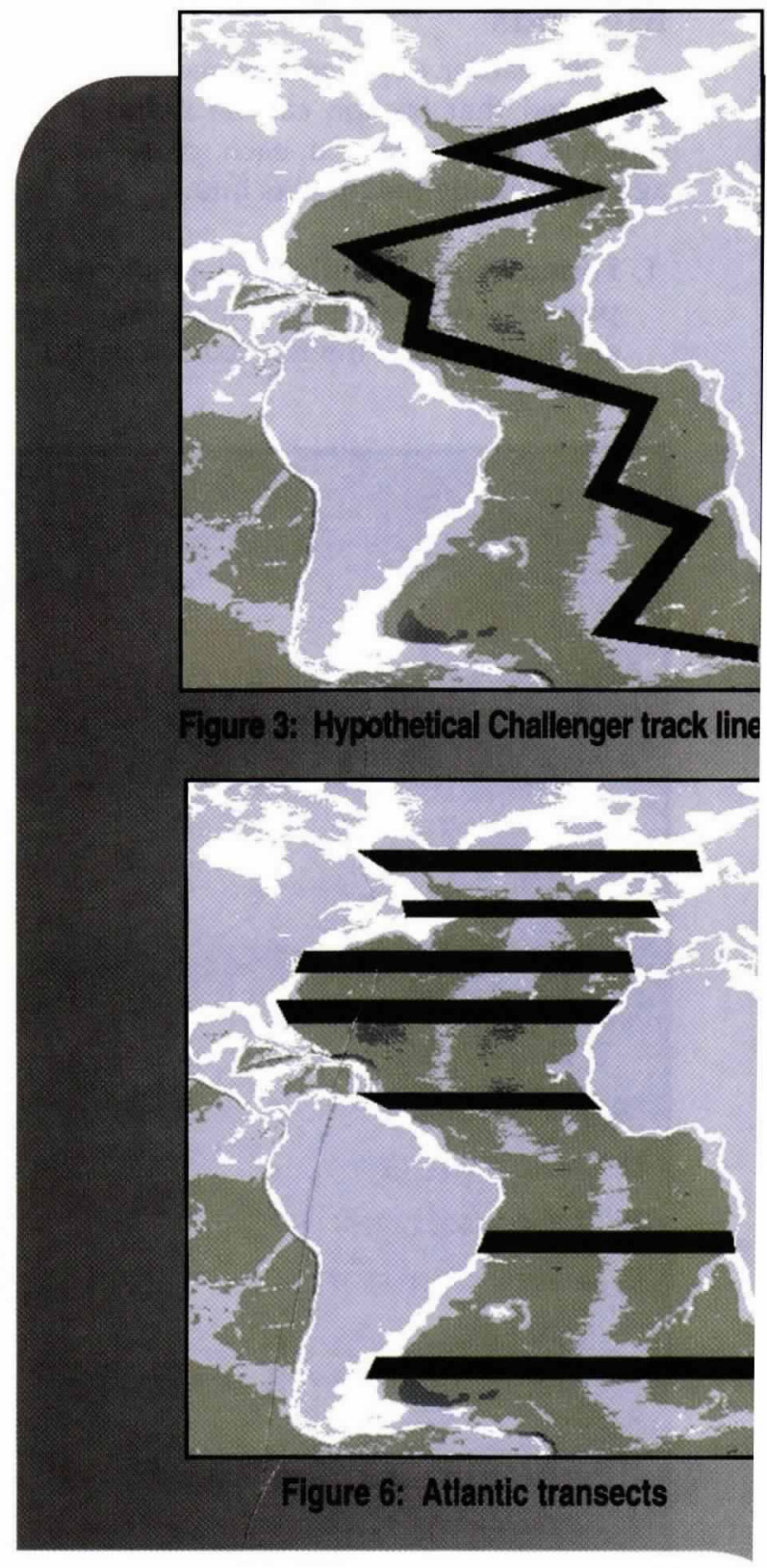

High Priority

Medium Priority

Low Priority
Figures 3-10. See text for description 


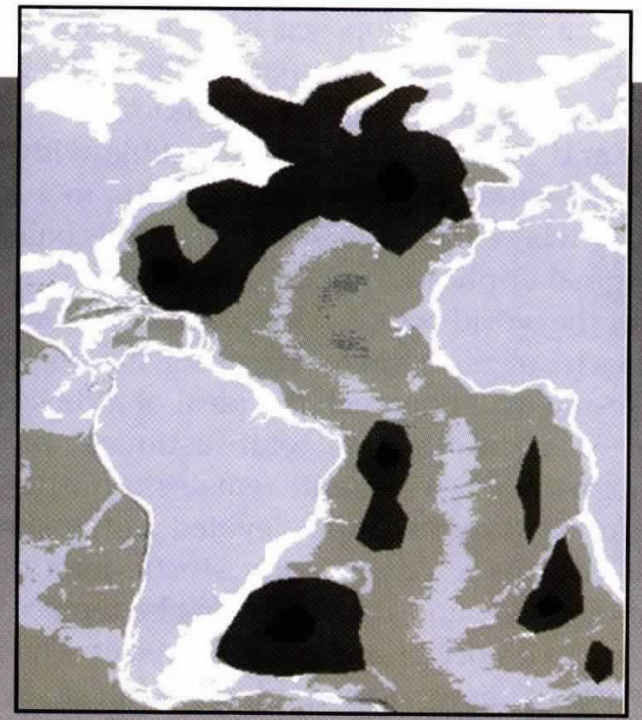

Figure 4: Deep oceanic survey requirements

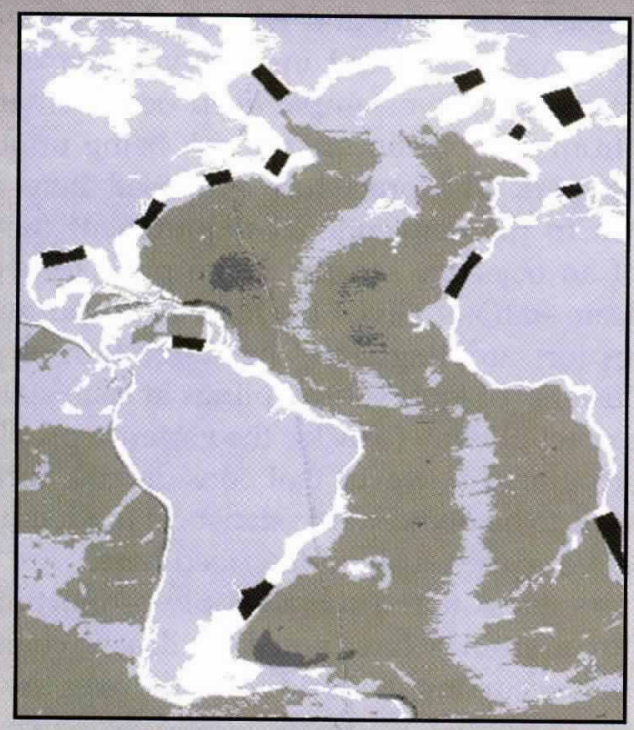

Figure 7: Sites of historical importance

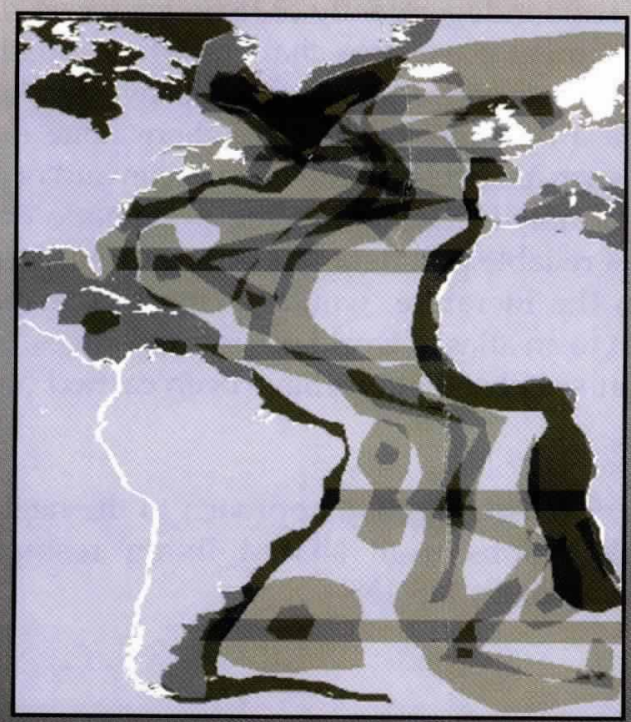

Figure 9: The composite joint probability of sampling taking Figures 3 to 8 into account

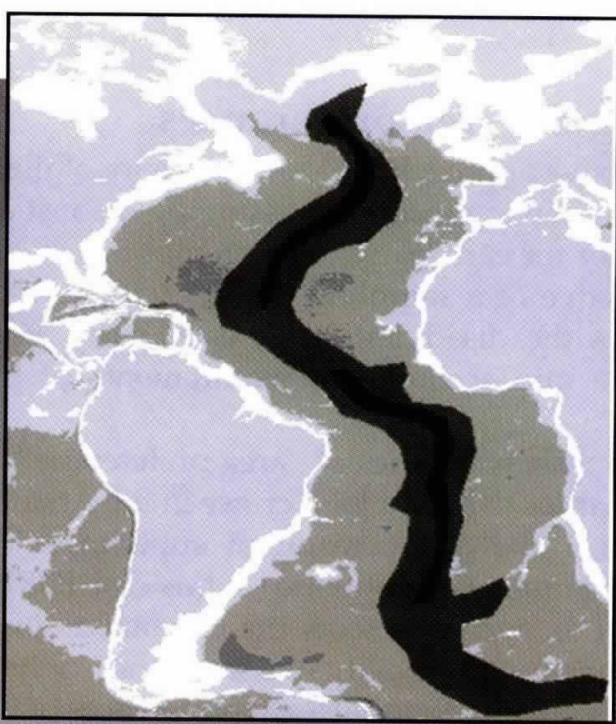

Figure 5: Mid-Atlantic ridge survey

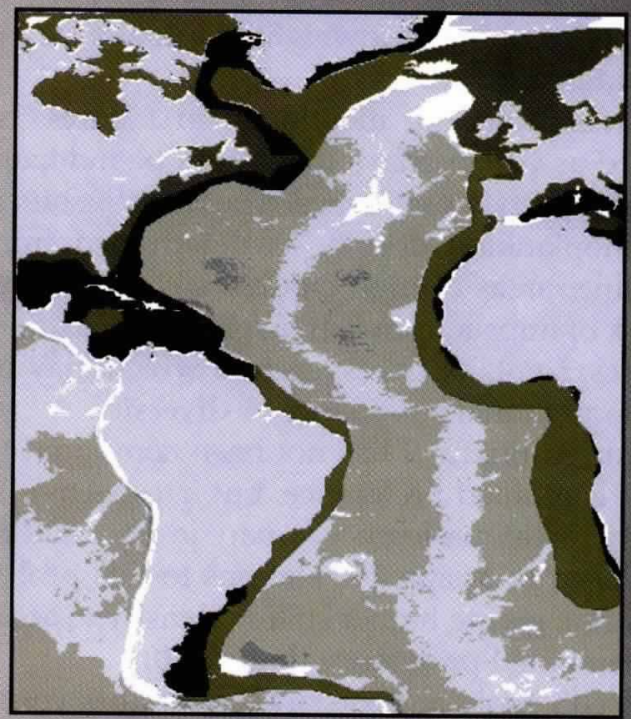

Figure 8: Continental shelf

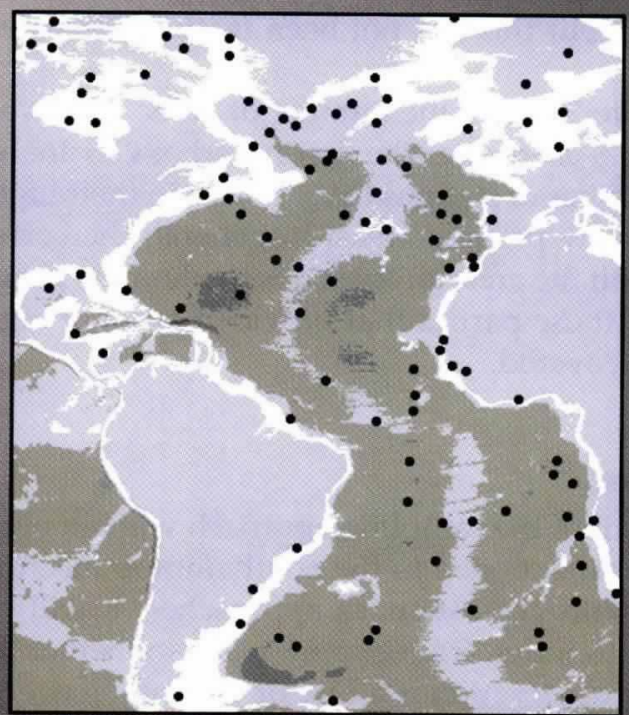

Figure 10: Stratified random sampling stations estimated by IDRISI 


\section{Results}

\section{Combining the area preferences}

Using the above approach (but for simplicity of illustration in the following examples assuming a constant weighting factor for each party), we attempt to achieve the basic objective of a sampling design /cruise track that minimizes the disadvantage to each party from having to take into account the requirements of the other parties.

Figures 3 to 8 show a series of "area preferences" of hypothetical parties to such a joint cruise in the Atlantic region. Figure 9 shows the result of superimposing these using a common GIS software (in this case IDRISI for Windows 1.0), and represents the combined "joint probability density/desirability of sampling" which represents the sum of individual areal preferences of the parties. In this case we have not assigned weightings to each of the 6 priority areas, and the plots simply represent the result of combining unweighted interests of the 6 parties considered.

Other considerations not mentioned in Figures 3-4 could be added as GIS overlays that might be independent of any one party's preference. These for instance, might be phytoplankton density as determined from remote sampling, areas of high biodiversity, areas subject to high risk of impact from fishing or eutrophication, transition zones, fronts or gyres; each of which may be indicators of local faunal abundance or diversity.

The sampling in Figure 9 has not been contoured to show areas of combined preference, but irrespective of this, the "joint areal preference" map in the IDRISI package was used to assign 200 stations to this probability distribution; giving higher station density to areas with higher combined preference (Figure 10).

The use of GIS tools and distance operators could also help in deciding on a cruise track that minimizes the total distance travelled depending on starting points chosen, but this has not been further presented here

Finally, Table 2 shows the number of "hits" achieved by each of the 6 users after the first round of GIS analysis: i.e. the number of stations that fell within their high, medium and low priority areas. In general, the correspondence with the areas proposed is moderately good; especially for those parties that provided areas of high priority to be covered.

\section{Conclusion}

One criticism of the sampling approach with an even spaced grid as mentioned in the Southampton meeting is that even with geostationary approaches, it will be very difficult to judge from survey results whether an occurrence or observation is a rare or a common event. Hence the idea of the Census as providing a 'total estimate' of number of fish present will be unlikely to be achieved. The approach proposed here does not necessarily provide an optimum alternative, but more closely resembles a random or stratified-random sampling framework, and perhaps is more compatible with post stratification. It certainly uses historical data in a more objective way where these are available, and is believed to be an original application of GIS procedures.

No area of the world oceans is excluded from the sampling approach suggested here. However the degree to which the results will approach the ideal of a census (in the sense of permitting a total estimate of the number/biomass of fish present) remains a function of the total effort and expenditure devoted to the survey. For various reasons a Census sensu strictu, may be an impractical objective, and could perhaps be replaced by the idea of an inventory of ecosystems/species. The procedure proposed here does perhaps offer a better prospect of completing such an inventory of unknown phenomena (assuming the specialists have some ideas of what remains to be known, and where), than a regular grid. A regular pattern would inevitably undersample interesting areas, and oversample deep ocean environments that from one preconception, risk being uniform for the most part. The procedure proposed here also approximates to the conventional requirement for the provision of an objective sample frame prior to beginning to allocate stations. Given the huge area and multiple priorities for sampling sites, the suggestion of performing "clusters" of local subsamplings at each station, may be one practical way to tackle the variance problem. This note recognizes however that considerable further attention needs to be dedicated to statistical modelling of mixed objective cruises such as described here.

The authors guess that a considerable proportion of the scientific community have noted the drastic changes that have been occurring in world fisheries over the last few decades. Fisheries scientists would also like the Census to contribute to characterizing further changes on a global scale independently of fisheries-based data. It could document ecosystem changes where possible, by comparing present results with past investigations, and provide a baseline for species where none exists presently - such as species of the deep ocean and oceanic ridges. As noted, assembling the systematic documentation of reliable past data sets now unpublished or scattered in the literature, would in itself be a major contribution to an atlas of world oceanic resources, even before any at-sea observations have been carried out.

\section{References}

Caddy, J.F. 1996: An objective approach to the negotiation of allocations from shared living resources. Marine Policy, 20(2), 145-155. 